\title{
Application of Raster to Vector Conversion in Power Electronics Thermal Infrared Images
}

\author{
Krastin Yordanov ${ }^{1}$, Iliya Hadzhidimov ${ }^{1}$, Peycho Popov ${ }^{2}$ \\ ${ }^{1}$ Technical University of Varna, Department of Thermal Engineering, 9010 Varna, Bulgaria \\ ${ }^{2}$ Technical University of Varna, Department of Mathematics and Physics, 9010 Varna, Bulgaria
}

\begin{abstract}
The infrared thermography today is widely used modern instrument for analysis of thermal fields in power electronics elements. On the other side, the heat fluxes causing temperature distribution of these elements and modules in working condition can be estimated in regimes of exploitation, while the cooling may be optimized in order to avoid damages of overheating. In present work, an algorithm and computer application for calculation temperature gradient on the surface of analyzed objects based on infrared thermal images is presented.
\end{abstract}

Keywords - infrared thermography, raster to vector conversion, heat dissipation, electronic elements.

\section{Introduction}

In engineering practice, today the thermal cameras are increasingly used. Recently, their prices were high but, today they are of better quality, more accessible and cheaper. Their application is almost everywhere and they are used in medicine, for military needs, civil engineering, electronics and many others to analyze processes connected with temperature distribution in wide ranges [1]. Due to permanent increasing of cameras precision in lot of cases there is a necessity to perform a detailed analysis of thermal image, which is usually saved in raster format on storage [2].

DOI: 10.18421/TEM92-01

https://doi.org/10.18421/TEM92-01

Corresponding author: Krastin Yordanov, Department of Thermal Engineering, Technical University of Varna, Varna, Bulgaria.

Email: krastin yordanov@tu-varna.bg

Received: 05 March 2020.

Revised: 02 April 2020.

Accepted: 08 April 2020.

Published: 27 May 2020.

(cc) BY-NC-ND (C) 2020 Krastin Yordanov, Iliya H adzhidimov \& Peycho Popov; published by UIKTEN. This work is licensed under the Creative Commons AttributionNonCommercial-NoDerivs 4.0 License.

The article is published with Open Access at www.temjournal.com
This paper includes a numerical procedure for transformation the raster image into a vector with opportunity to determine the temperature on a pixel level when this image has been loaded on a personal computer. In this case, vectorization means to transform raster image in a numerical format - every pixel of the image corresponds to a temperature value and base on this matrix of temperatures, visualization with different sizes may be realized without loss of quality. The average integral temperature value of different image regions and the temperature gradient field in 2D Cartesian system of the image are estimated. An application intended for managing infrared thermal images made with camera Flir i7 is developed. The application may be adopted to work with any different thermal camera with availability of color band for visualization of corresponding temperatures with presence of minimal and maximal temperature value on the picture. The temperature gradient can give very important information about the direction [3] of thermal flux in the power electronics systems and elements. This may be used to realize optimal and effective cooling of most of the thermal loaded objects or the perform optimization of cooling facilities. The proposed application includes algorithm for digitalizing the raster thermal image of the infrared camera Flir i7, as every pixel of the image has been transformed into a digital value of correspondent to the screen pixel temperature. After this, a matrix of temperature values is created and another one with the values of temperature gradient module [4].

Within the software application, these procedures are visualized in order to specify the zones of the treated object with maximal thermal load. It is possible the numerical image to be smoothen. As a result, both matrixes created may be used for detailed analysis of the thermal behavior of the object, to find the area with maximal temperature and gradient on a pixel level [5].

The examples demonstrated are raster images in JPG format made with thermal camera Flir i7 and they are with dimension $240 \times 240$ pixels. Basically, these examples are connected with electronic elements with different level of thermal load. 
In addition, the present development aims to estimate heat quantity dissipated from the part of surface of the treated body assuming thermal convection and radiation from the electronics surfaces as a heat source.

\section{Mathematical Background}

To realize the development, a spline procedure for interpolation/smoothing is used [6], [7]. The idea is to implement superposition method upon every color pixel of the raster image for the temperature from the camera usually in JPG format. The numerical values for every pixel are calculated by a cubic spline function [8]. The cubic spline consists of polynomial order of third degree, Figure 1. The number of these polynomials is determined by the control points, containing the function in numerical way.

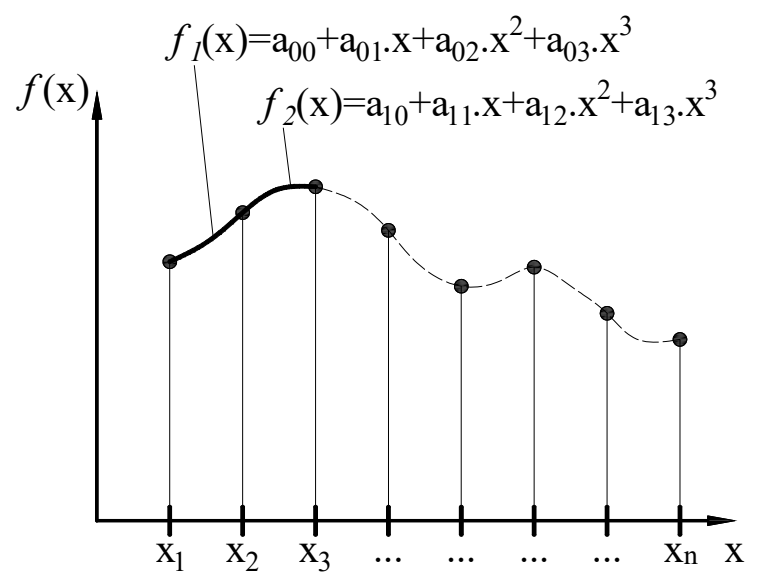

Figure 1. Cubic ,spline“ function

The second derivative of every polynomial has value zero at the ends of control points. From this point of view, this polynomial is called "natural" end leads to tridiagonal system of linear equations which decision gives the coefficients of the different polynomials for every interval. Actually, these splines may be used for interpolation and smoothing, for obtaining integral and derivative values of the function.

The spline procedure in algorithm proposed consecutively finds temperature values in every pixel of the image horizontally, after which the values are one more recalculated in vertical order, Figure 2. Values calculated after second spline procedure is the numerical conformity of the raster image. The procedure proposed uses so called "smoothing spline" [9], which gives the opportunity not only to interpolate the data, but to perform smoothing with a constant or individual coefficient for every interval of control points [10]. In present paper, a constant coefficient of interpolation/smoothing for all the intervals has been implemented.

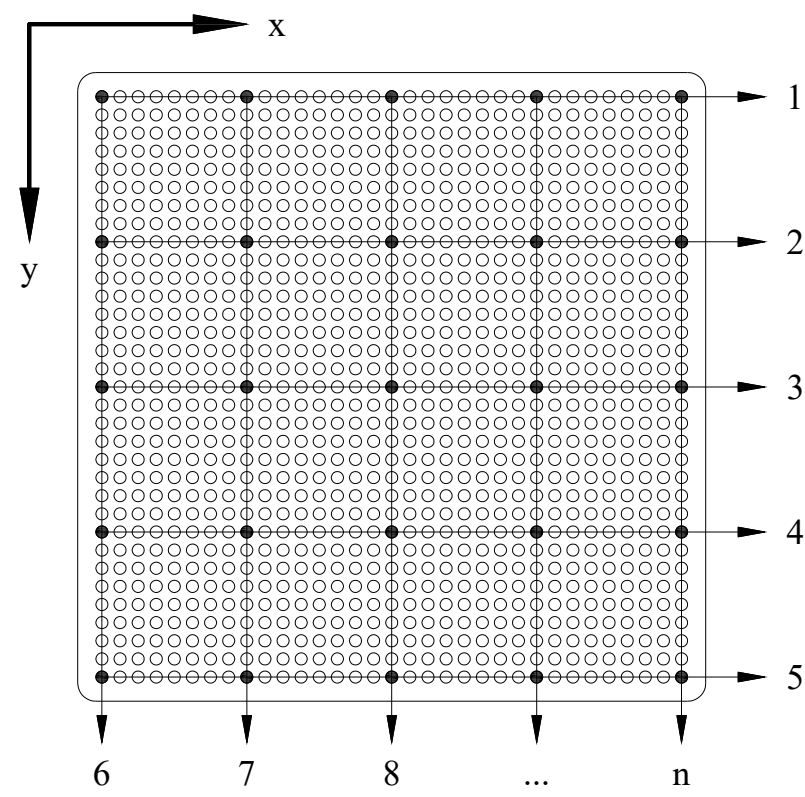

Figure 2. Scanning pixels from the image (computer screen) in horizontal and vertical direction. Black points are from the raster image (240x240), white are derived from the spline function as internal values for the control points intervals.

After every procedure has finished, temperature of every pixel of the image is described by a polynomial of third degree:

$$
t(p)=\mathrm{a}_{0}+\mathrm{a}_{1} \cdot x+\mathrm{a}_{2} \cdot x^{2}+\mathrm{a}_{3} \cdot x^{3},
$$

in which

$t(x)$ - Temperature, ${ }^{\circ} \mathrm{C}$;

$\mathrm{a}_{i}(i=0,1,2,3)$ - Cubic spline coefficients;

$p$ - Argument with values in dependence of geometry of the pixel in 2D Cartesian coordinate system of the image, $p=p(x, y)$.

The polynomials of third degree are describing with good precision and enough accurately the behavior of the function (temperature), and they have advantages to be smooth function which may be integrated or to find its first derivative without any singularity [11]. In this case, the pixels scanning procedure of the image is performed twice - in horizontal and vertical direction to find the interpolated in 2D temperature value [12]. The advantage of the method is in opportunity to find and the temperature gradient value in every point of interpolation together with the potential (temperature). The temperature gradient in $\mathrm{x}-\mathrm{y}$ can be obtained after differentiation the equation (1):

$$
\begin{aligned}
& \frac{d t(p)}{d x}=a_{1}+a_{2} \cdot p+a_{3} \cdot p x^{2} \\
& \frac{d t(p)}{d y}=a_{1}^{\prime}+a_{2}^{\prime} \cdot p+a_{3}^{\prime} \cdot p^{2}
\end{aligned}
$$

in which 
$q(p)$ - Module of the gradient, ${ }^{\circ} \mathrm{C} / \mathrm{m}$;

$a_{i}$ and $a_{1}^{\prime}(i=1,2,3)$ - Cubic spline coefficients; $x, y-$ Point arguments position in $2 \mathrm{D}$.

The module of the temperature gradient is as follows:

$$
q(p)=\sqrt{\left(\frac{d t(p)}{d x}\right)^{2}+\left(\frac{d t(p)}{d y}\right)^{2}}
$$

The algorithm may be demonstrated on infrared raster image of Peltier element in JPG format, Figure 3 , loading raster image and order of data treatment in computer application:

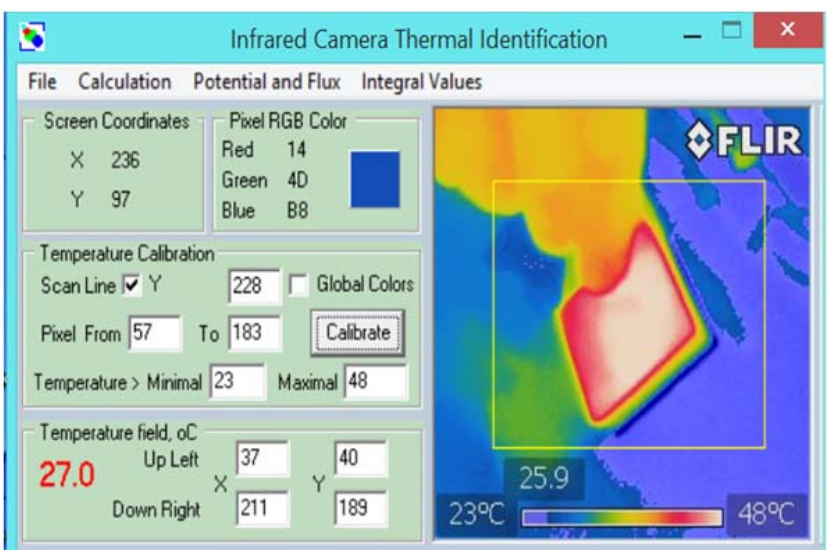

Figure 3. Infrared picture of Peltier element with dimension 240x240 pixels

After loading the image, the first step is to find the correspondence between color code table in RGB and temperature. Scanning the color band at the bottom of the picture helps to associate the color table linearly with minimal and maximal temperature values $\left(23^{\circ} \mathrm{C}\right.$ and $\left.48^{\circ} \mathrm{C}\right)$. After the association of colors and temperatures, the part of the image for thermal analysis has been marked. In Figure 3, this is the rectangle area between yellow lines and in Figure 4 , and the order of operations with the raster image may be seen.

The next step is to perform two times spline interpolation/smoothing in horizontal and vertical direction. As a result, the matrix of temperatures for chosen dimensions in $\mathrm{X}$ and $\mathrm{Y}$ has been obtained. So the raster image is transformed into digital - from colors to temperature values for any point. In Figure 5 , the picture of smoothed temperature filed is shown.

The spline function allows choosing between interpolations and smoothing by the help of parameter which may be different for any spline interval. The value of this coefficient is set to constant for all the points and its value varies from $1.10^{10}$ for interpolation to 1 for smoothing. The digitalized picture consists of 25 equipotential temperature surfaces.

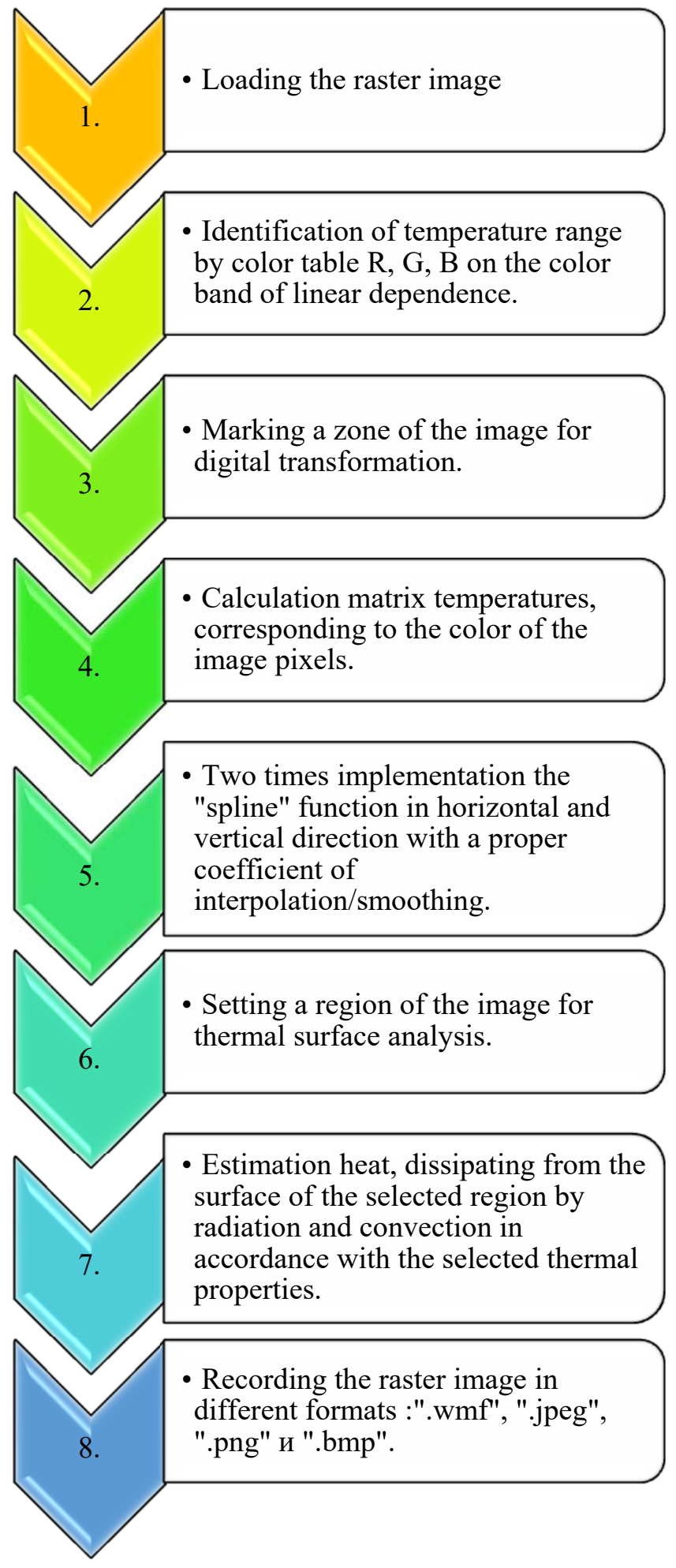

Figure 4. Order of infrared image treatment

The right side of Figure 6 contains zones in white leading to high gradients, where the heat is dissipated most intensively in the ambient. Other opportunity, which may be successfully implemented, is to use digitalized raster image to calculate the heat dissipated by radiation and convection from the surface of the power electronic elements. 


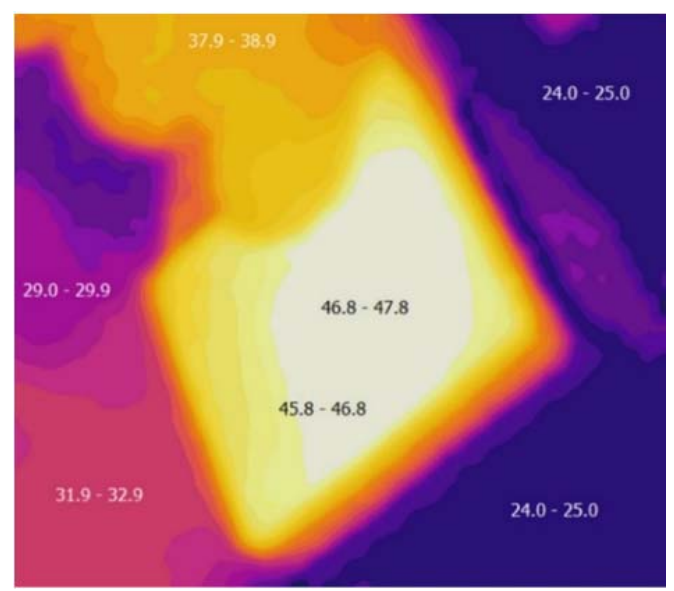

Figure 5. Smoothed picture of temperature field based on the matrix of points temperatures

By above mention procedure the gradient temperature field is also calculated and shown in Figure 6.

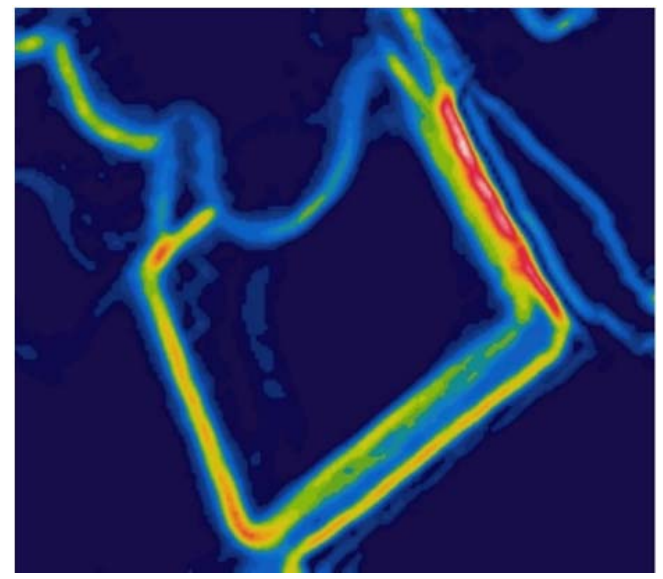

Figure 6. Picture of temperature gradient in X-Y surface

The algorithm allows assuming average or average integral value of temperature of the part of the image and dissipated heat [13], [14] if some thermal properties of the object are known:

$$
\begin{gathered}
E=E_{r a d}+E_{c o n v} \\
E_{r a d}=\varepsilon \cdot c_{s} \cdot\left[\left(\frac{T_{o b j}}{100}\right)^{4}-\left(\frac{T_{a m b}}{100}\right)^{4}\right] \cdot A \\
E_{c o n v}=h_{c} \cdot\left(T_{o b j}-T_{a m b}\right) \cdot A,
\end{gathered}
$$

in which

$E$ - Heat from surface of the object to the ambient, $\mathrm{W}$;

$E_{\text {rad }}$ - Radiative component of the heat, W;

$E_{\text {conv }}$-Convective component of the heat, W;

$\varepsilon-$ Emissivity of the surface material;

$c_{S}=5,67$, emissivity of "Black body", $\mathrm{W} /\left(\mathrm{m}^{2} \cdot \mathrm{K}^{4}\right)$;

$T_{o b j}$ - Temperature of the object's surface, $\mathrm{K}$;

$T_{a m b}$ - Ambient temperature, $\mathrm{K}$;

$A$ - Area of the treated surface, $\mathrm{m}^{2}$; $h_{c}$ - Convective heat transfer coefficient, $\mathrm{W} /\left(\mathrm{m}^{2} \cdot \mathrm{K}^{4}\right)$.

Average integral value of the temperature is calculated by integration of the temperature on area, and this area has been discretized in fixed number triangle elements on which area the numerical integration is made [15]. In most of the real cases, the average value coincides with the average integral. Difference may be observed in cases when the object has very high temperature gradient in $\mathrm{X}-\mathrm{Y}$.

\section{Experiment Realization}

The method has been implemented on some electrical devices in order to calculate the heat from the active surface.

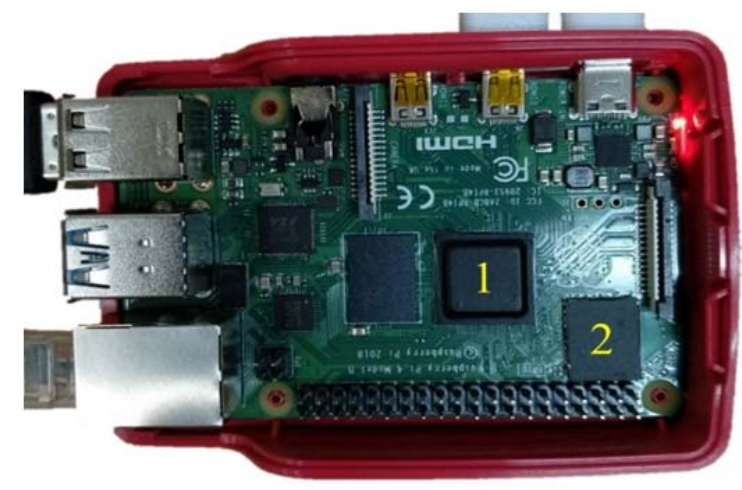

Figure 7. Picture of Raspberry Pi 4

In Figure 7, a picture of widely used platform Raspberry Pi 4, and in Figure 8, the corresponding infrared image can be seen. To avoid distortion of the temperature filed in two regions marked with 1 and 2 , where the electronics is under metal cover with low surface emissivity, the same surfaces has been supplied with dark math covers with emissivity of about 0,95 . This makes possible to measure the temperature of the object with precision about $\pm 0,2^{\circ} \mathrm{C}$. The temperature of $-19,7^{\circ} \mathrm{C}$ in the figure is the fixed minimal temperature of the camera.

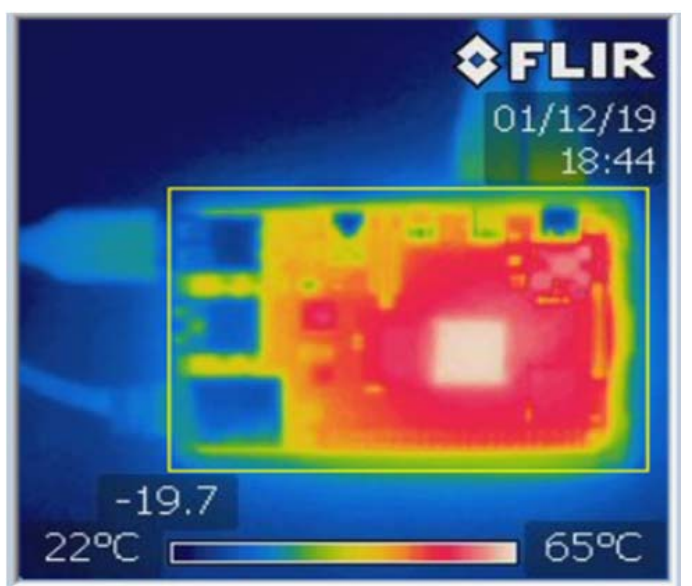

Figure 8. Raw image of temperature filed in working condition of Raspberry Pi 4 with marked region for analysis 
After the treatment of infrared image has finished, a digital version of the raster is derived, Figure 9 and the gradient, Figure 10.

After fixing the correspondence values between real dimensions of the module and pixels of the screen image, the dissipated heat from the surface may be calculated, this is shown in Figure 11. Average integral temperature of the selected area is $49,6^{\circ} \mathrm{C}$, and total heat quantity from the surface is about $2 \mathrm{~W}$, radiation part is $0,94 \mathrm{~W}$ and $1,05 \mathrm{~W}$ convective.

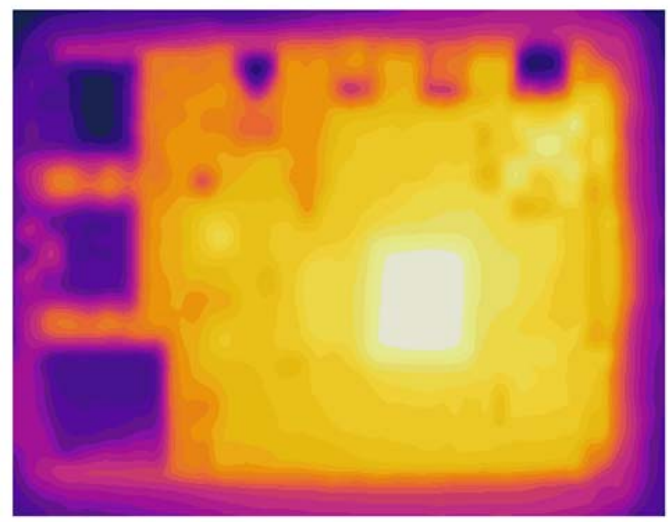

Figure 9. Digital image of the temperature field in working condition of Raspberry Pi 4 (fragment)

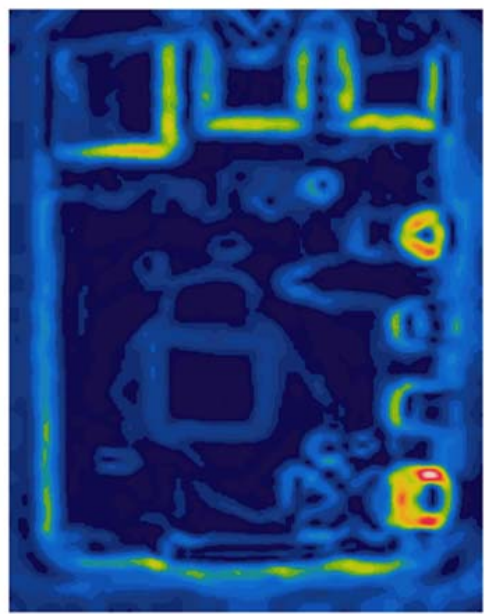

Figure 10. Temperature gradient of the selected fragment of the image. Bright zones are with highest gradient values.
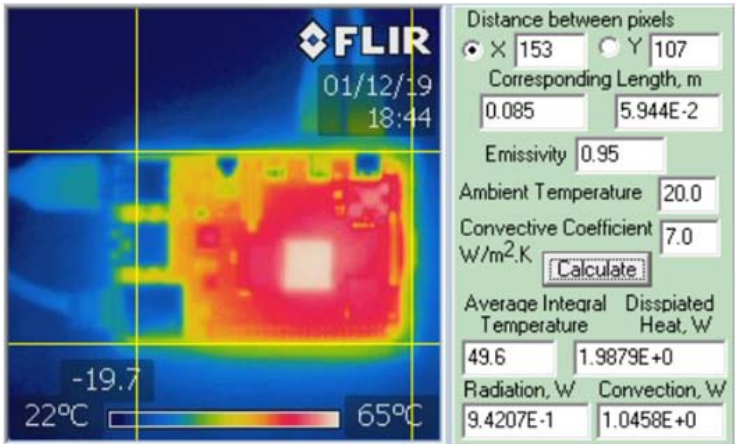

Figure 11. Dissipated heat from the selected surface, radiation and convection
In Figure 12, the numerical image of the fragment around processors area where the temperature is highest is presented. The different isopotential surfaces describe zones with equal temperatures with temperature difference of $0,4^{\circ} \mathrm{C}$ within the surfaces.

The following conversion of raster to vector image is for voltage regulator XH-M401. In Figure 13 and Figure 14, the regulator and its raster image are shown in steady state working condition.

The same procedure as above mentioned is performed and in Figure 15, the result of vectorization of the temperature field may be seen. With a cross, the position with maximum temperature is noted.

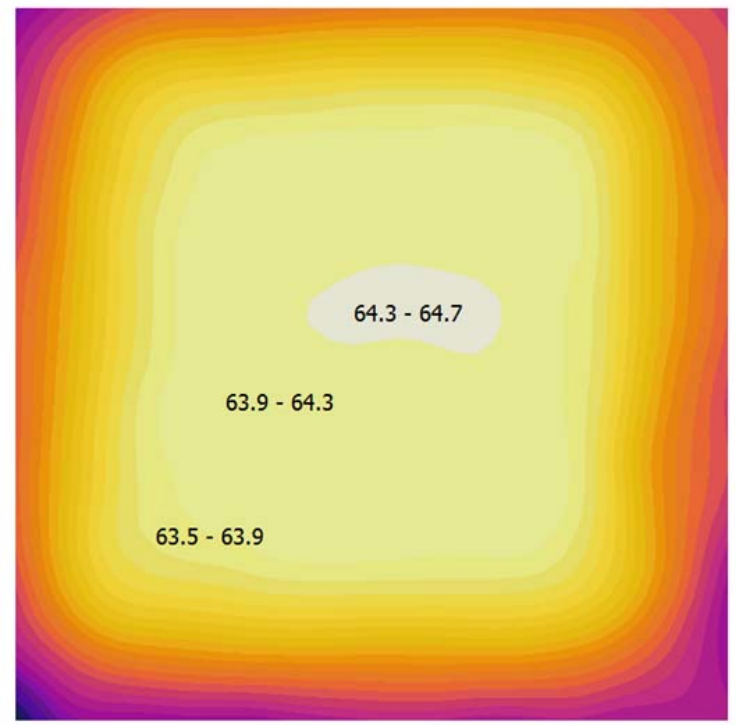

Figure 12. Digitalized image of the processor temperature field

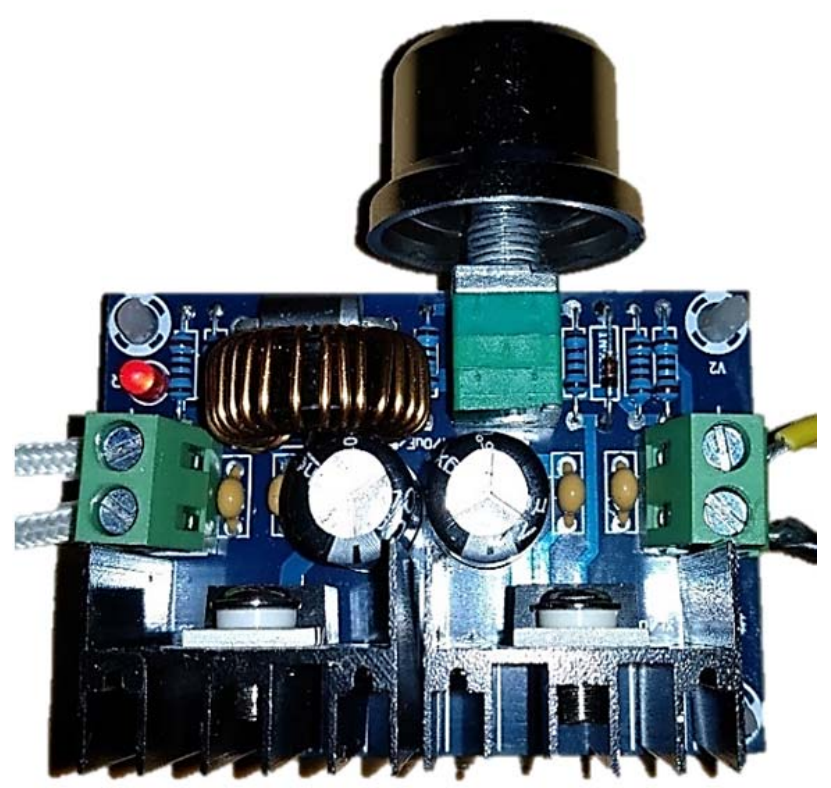

Figure 13. Voltage regulator $\mathrm{XH}-\mathrm{M} 401$ 


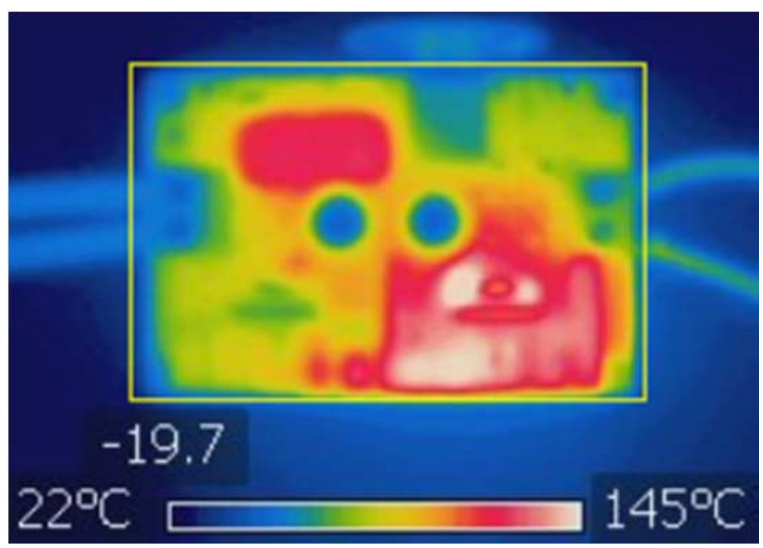

Figure 14. Raster image of XH-M401, fragment

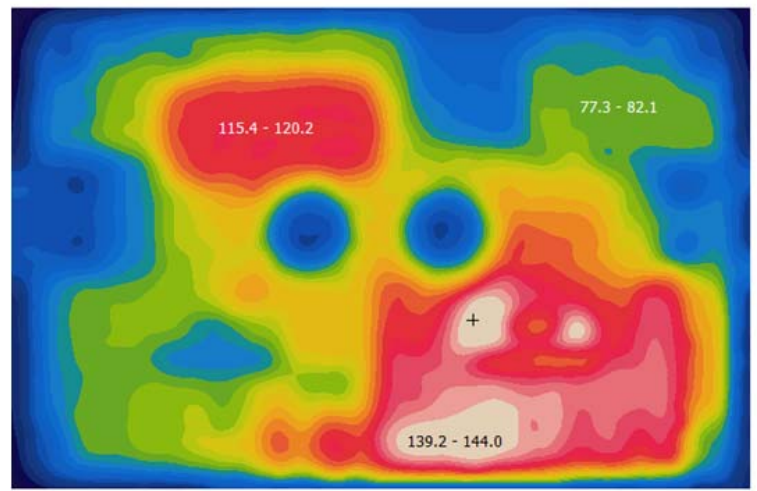

Figure 15. Numerical format image of the infrared picture of XH-M401

Figure 15 show that only one channel is loaded and in that case through the radiator passes the main quantity of heat. In the zone of contact with the base, without frames maximum value of temperature has been observed. In Figure 16, the gradient field is visualized. Logically, the highest gradient value is through radiator.

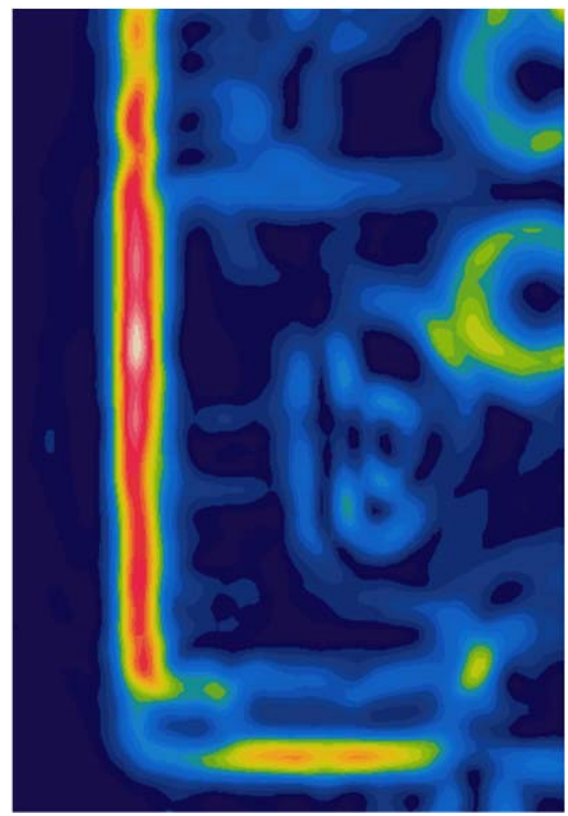

Figure 16. Temperature gradient field
The last equipment examined is a flat electric heater. In Figure 17 and Figure 18, the image of the object and zoomed fragment of the raster image made by infrared camera are shown.

The method proposed is implemented to this object in order to calculate the heat spread by radiation and convection from the visible surface of the heater.

In Figure 19, the treated temperature field can be seen and in Figure 20, heat calculated from the active surface. In this figure, some values of equipotential temperature zones are printed.

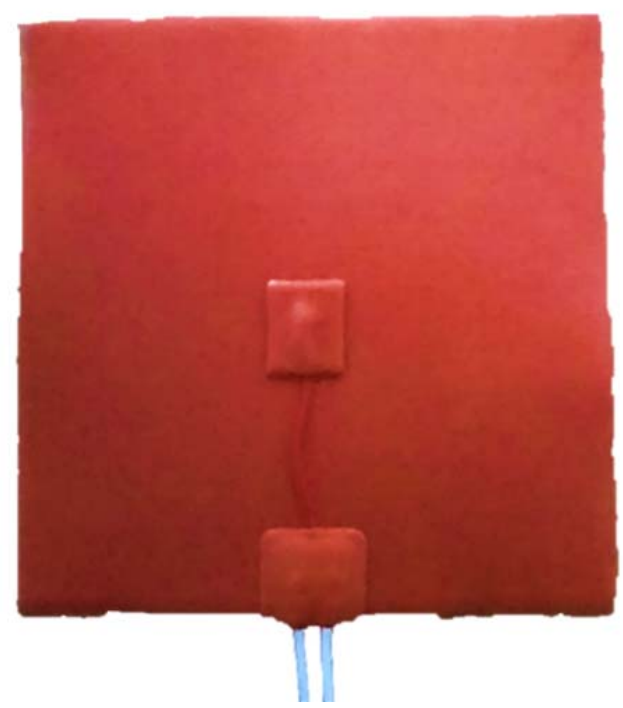

Figure 17. Picture of a flat electric heater for $3 D$ printer

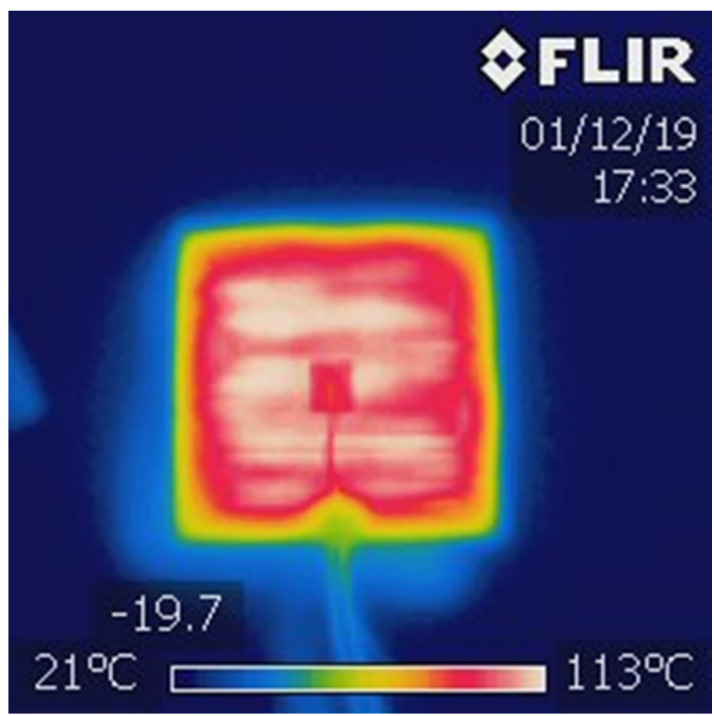

Figure 18. Raw raster Flir i7 image of the heater

Having in mind the heater dimensions of $20 \times 20$ $\mathrm{cm}$, we may calculate the heat fluxes from this surface when the ambient temperatures is $20^{\circ} \mathrm{C}$, emissivity of the surface is 0,95 and convective heat transfer coefficient is $7 \mathrm{~W} /\left(\mathrm{m}^{2} . \mathrm{K}\right)$. In Figure 20 , the power of radiative and convective heat fluxes may be seen. Their values are $22,9 \mathrm{~W}$ and $20,5 \mathrm{~W}$ for the 
radiative and convective component respectively. So the total heat power of the visible side of the heater is $43,4 \mathrm{~W}$ by average integral temperature value of surface $92,7^{\circ} \mathrm{C}$. In Figure 21 the generated vector image with isolines made by splines in Autodesk AutoCAD is presented. This image has been built by importing the numerical temperature matrix after raster to digital conversion.

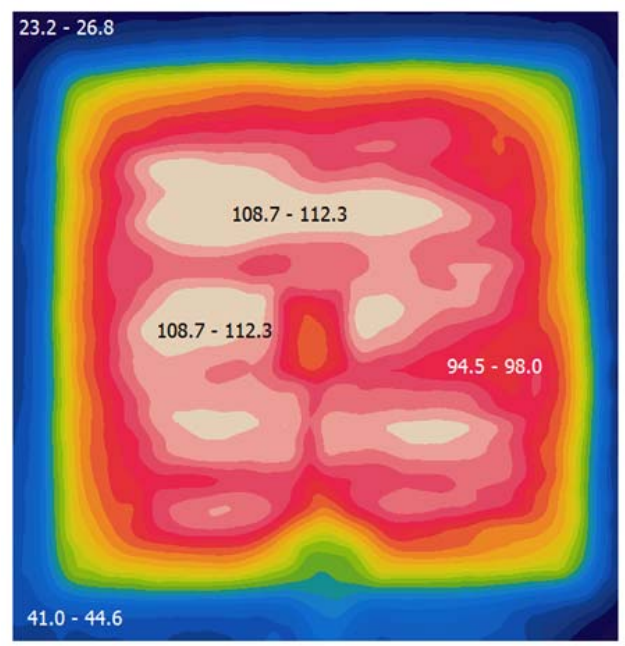

Figure 19. Digital temperature field of the visible side of the heater

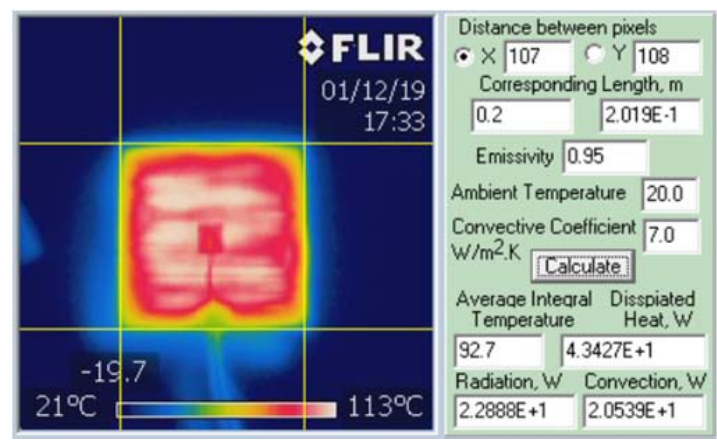

Figure 20. Calculated values of the dissipated heat by components and totally from the visible active side of the heater

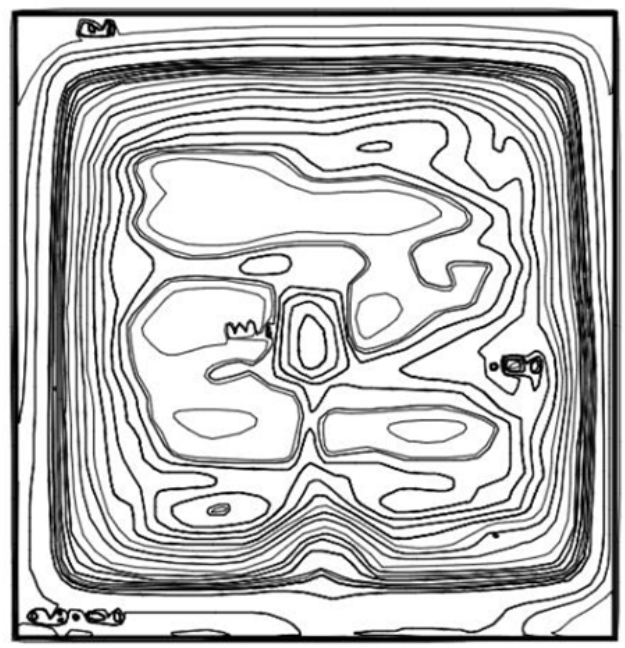

Figure 21. Isolines based on infrared image of the heater in AutoCAD

\section{Conclusion}

When analyzing the thermal infrared images connected with temperature fields of power electronics elements, it is possible successfully to implement conversion of raster image into a digital one. This may help to have a detailed and precise picture of important parts of the object appropriate for normal functioning of these elements, monitoring and improving cooling facilities. When calculating both components of the heat dissipated from the active surfaces - radiative and convective, we have to have in mind that the exact or correct values of some important thermal properties have to be known. In this number emissivity of the surfaces and especially convective heat transfer coefficient in a high degree are main factors for heat correct calculation. Also knowing these parameters, it may be very useful when building a numerical model of the treated bodies with Finite Elements Analysis.

\section{Acknowledgements}

The paper has been developed in the frames of the project "Model Based Design of Power Electronic Devices with Guaranteed parameters", ДH07/6/15.12.2016, BNSF.

\section{References}

[1]. Ellison, G. (2010). Thermal computations for electronics: conductive, radiative, and convective air cooling. CRC press.

[2]. Remsburg, R. (2011). Advanced thermal design of electronic equipment. Springer Science \& Business Media.

[3]. Bormashov, V. S., Tarelkin, S. A., Buga, S. G., Kuznetsov, M. S., Terentiev, S. A., Semenov, A. N., \& Blank, V. D. (2013). Electrical properties of the high quality boron-doped synthetic single-crystal diamonds grown by the temperature gradient method. Diamond and related materials, 35, 19-23.

[4]. Tasdemir, S., Urkmez, A., \& Inal, S. (2011). Determination of body measurements on the Holstein cows using digital image analysis and estimation of live weight with regression analysis. Computers and electronics in agriculture, 76(2), 189-197.

[5]. Lingling, G., Rongqun, Z., Ming, Z., Hui, Y., \& Simin, C. (2009). Design and implementation of soil spatial variation analysis system. WSEAS Transactions on Information Science and Applications, 6(7), 1135-1144.

[6]. Mazzola, G., Milmeister, G., \& Weissmann, J. (2006). Comprehensive Mathematics for Computer Scientists 2: Calculus and ODEs, Splines, Probability, Fourier and Wavelet Theory, Fractals and Neural Networks, Categories and Lambda Calculus. Springer.

[7]. Weinert, H. L. (2013). Fast compact algorithms and software for spline smoothing. SpringerBriefs in Computer Science. New York: Springer. 
[8]. McCartin, B. J. (1991). Theory of exponential splines. Journal of Approximation Theory, 66(1), 123.

[9]. Mohammadi, R. (2013). Exponential B-spline solution of convection-diffusion equations. Applied Mathematics, 4, 933-944.

[10]. Dag, I., \& Ersoy, O. (2016). The exponential cubic B-spline algorithm for Fisher equation. Chaos, Solitons \& Fractals, 86, 101-106.

[11]. Shang, D. Y. (2013). Free Convection Film Flows and Heat Transfer: Models of Laminar Free Convection with Phase Change for Heat and Mass Transfer Analysis. Springer Science \& Business Media.
[12]. Martynenko, O. G., \& Khramtsov, P. P. (2005). Free-convective heat transfer: with many photographs of flows and heat exchange. Springer Science \& Business Media..

[13]. Kurazumi, Y., Rezgals, L., \& Melikov, A. K. (2014). Convective heat transfer coefficients of the human body under forced convection from ceiling. Journal of Ergonomics, 4(1), 1000126.

[14]. Feng, S., Shi, M., Yan, H., Sun, S., Li, F., \& Lu, T. J. (2018). Natural convection in a cross-fin heat sink. Applied Thermal Engineering, 132, 30-37.

[15]. Teutan, E., Bara, M., \& Ardelean, I. (2010). Theoretical considerations regarding the virtual modelling of surfaces used in topography. Mechanics, 85(5), 61-65. 